DOI: $10.15393 /$ j3.art.2016.3010

UDC 517.54

\author{
D. Vamshee Krishna, T. RamReddy
}

\title{
COEFFICIENT INEQUALITY FOR MULTIVALENT BOUNDED TURNING FUNCTIONS OF ORDER $\alpha$
}

\begin{abstract}
The objective of this paper is to obtain the sharp upper bound to the $H_{2}(p+1)$, second Hankel determinant for $p$-valent (multivalent) analytic bounded turning functions (also called functions whose derivatives have positive real parts) of order $\alpha(0 \leq \alpha<1)$, using Toeplitz determinants. The result presented here includes three known results as their special cases.
\end{abstract}

Key words: p-valent analytic function, bounded turning function, upper bound, Hankel determinant, positive real function, Toeplitz determinants

2010 Mathematical Subject Classification: 30C45, 30C50

1. Introduction. Let $A_{p}$ denote the class of functions $f$ of the form

$$
f(z)=z^{p}+a_{p+1} z^{p+1}+\cdots
$$

in the open unit disc $E=\{z:|z|<1\}$ with $p \in \mathbb{N}=\{1,2,3, \ldots\}$. Let $S$ be the subclass of $A_{1}=A$, consisting of univalent functions.

In 1985, Louis de Branges de Bourcia proved the Bieberbach conjecture, i.e., for a univalent function its $n^{\text {th }}$ coefficient is bounded by $n$ (see [1]). The bounds for the coefficients of these functions give information about their geometric properties. In particular, the growth and distortion properties of a normalized univalent function are determined by the bound of its second coefficient. The Hankel determinant of $f$ for $q \geq 1$ and $n \geq 1$ was defined by Pommerenke [2] as

$$
H_{q}(n)=\left|\begin{array}{cccc}
a_{n} & a_{n+1} & \cdots & a_{n+q-1} \\
a_{n+1} & a_{n+2} & \cdots & a_{n+q} \\
\vdots & \vdots & \vdots & \vdots \\
a_{n+q-1} & a_{n+q} & \cdots & a_{n+2 q-2}
\end{array}\right| .
$$


This determinant has been considered by several authors in the literature. For example, Noonan and Thomas [3] studied the second Hankel determinant of areally mean $p$-valent functions. Noor [4] determined the rate of growth of $H_{q}(n)$ as $n \rightarrow \infty$ for functions in $S$ with bounded boundary rotation. The Hankel transform of an integer sequence and some of its properties were discussed by Layman [5]. One can easily observe that the Fekete-Szegö functional is $H_{2}(1)$. Fekete-Szegö then further generalized the estimate $\left|a_{3}-\mu a_{2}^{2}\right|$ with real $\mu$ and $f \in S$. Ali [6] found sharp bounds on the first four coefficients and sharp estimate for the Fekete-Szegö functional $\left|\gamma_{3}-t \gamma_{2}^{2}\right|$, where $t$ is real, for the inverse function of $f$ for $p=1$, given in (1.1), defined as $f^{-1}(w)=w+\sum_{n=2}^{\infty} \gamma_{n} w^{n}$, when $f \in \widetilde{S T}(\alpha)$, the class of strongly starlike functions of order $\alpha(0<\alpha \leq 1)$. Further sharp bounds for the functional $\left|a_{2} a_{4}-a_{3}^{2}\right|$, the Hankel determinant in the case of $q=2$ and $n=2$, known as the second Hankel determinant (functional), given by

$$
H_{2}(2)=\left|\begin{array}{ll}
a_{2} & a_{3} \\
a_{3} & a_{4}
\end{array}\right|=a_{2} a_{4}-a_{3}^{2},
$$

were obtained for various subclasses of univalent and multivalent analytic functions by several authors in the literature. Janteng et al. [7] have considered the functional $\left|a_{2} a_{4}-a_{3}^{2}\right|$ and found a sharp upper bound for the function $f$ in the subclass $\mathcal{R}$ of $S$, consisting of functions whose derivative has a positive real part (also called bounded turning functions) studied by MacGregor [8]. In their work, they have shown that if $f \in \mathcal{R}$ then $\left|a_{2} a_{4}-a_{3}^{2}\right| \leq \frac{4}{9}$. Motivated by this result, in this paper we consider the Hankel determinant in the case of $q=2$ and $n=p+1$, denoted by $H_{2}(p+1)$, given by

$$
H_{2}(p+1)=\left|\begin{array}{ll}
a_{p+1} & a_{p+2} \\
a_{p+2} & a_{p+3}
\end{array}\right|=a_{p+1} a_{p+3}-a_{p+2}^{2} .
$$

Further, we seek a sharp upper bound to the functional $\left|a_{p+1} a_{p+3}-a_{p+2}^{2}\right|$ for the functions belonging to the certain subclass of $p$-valent analytic functions, defined as follows.

Definition 1. A function $f(z) \in A_{p}$ is said to be in the class $\mathcal{R}_{p}(\alpha)$ $(0 \leq \alpha<1)$ if it satisfies the condition

$$
R e \frac{f^{\prime}(z)}{p z^{p-1}}>\alpha, \quad \forall z \in E .
$$


1) If $p=1$, we obtain $\mathcal{R}_{1}(\alpha)=\mathcal{R}(\alpha)$, the class of bounded turning functions of order $\alpha$.

2) Choosing $\alpha=0$, we get $\mathcal{R}_{p}(0)=\mathcal{R}_{p}$, the class of $p$-valent bounded turning functions.

3) Selecting $p=1$ and $\alpha=0$, we have $\mathcal{R}_{1}(0)=\mathcal{R}$.

In the next section we give some preliminary Lemmas required for proving our result.

2. Preliminary Results. Let $\mathcal{P}$ denote the class of functions consisting of $g$ such that

$$
g(z)=1+c_{1} z+c_{2} z^{2}+c_{3} z^{3}+\ldots=1+\sum_{n=1}^{\infty} c_{n} z^{n},
$$

which are regular in the open unit disc $E$ and satisfy $\operatorname{Re} g(z)>0$ for any $z \in E$. Here $g(z)$ is called a Caratheòdory function [9].

Lemma 1. [10, 11] If $g \in \mathcal{P}$, then $\left|c_{k}\right| \leq 2$, for each $k \geq 1$ and the inequality is sharp for the function $\frac{1+z}{1-z}$.

Lemma 2. [12] The power series for $g(z)=1+\sum_{n=1}^{\infty} c_{n} z^{n}$ given in (6) converges in the open unit disc $E$ to a function in $\mathcal{P}$ if and only if the Toeplitz determinants

$$
D_{n}=\left|\begin{array}{ccccc}
2 & c_{1} & c_{2} & \cdots & c_{n} \\
c_{-1} & 2 & c_{1} & \cdots & c_{n-1} \\
c_{-2} & c_{-1} & 2 & \cdots & c_{n-2} \\
\vdots & \vdots & \vdots & \vdots & \vdots \\
c_{-n} & c_{-n+1} & c_{-n+2} & \cdots & 2
\end{array}\right|, n=1,2,3 \ldots
$$

and $c_{-k}=\bar{c}_{k}$, are all non-negative. They are strictly positive except for $p(z)=\sum_{k=1}^{m} \rho_{k} p_{0}\left(e^{i t_{k}} z\right)$, with $\sum_{k=1}^{m} \rho_{k}=1, t_{k}$ real and $t_{k} \neq t_{j}$, for $k \neq j$, where $p_{0}(z)=\frac{1+z}{1-z}$; in this case $D_{n}>0$ for $n<(m-1)$ and $D_{n} \doteq 0$ for $n \geq m$.

This necessary and sufficient condition found in [12] is due to Caratheòdory and Toeplitz. We may assume without restriction that $c_{1}>0$. From Lemma 2, for $n=2$ we have

$$
D_{2}=\left|\begin{array}{ccc}
2 & c_{1} & c_{2} \\
\bar{c}_{1} & 2 & c_{1} \\
\bar{c}_{2} & \bar{c}_{1} & 2
\end{array}\right|
$$


On expanding the determinant, we get

$$
D_{2}=8+2 \operatorname{Re}\left\{c_{1}^{2} c_{2}\right\}-2\left|c_{2}\right|^{2}-4\left|c_{1}\right|^{2} \geq 0 .
$$

Applying the fundamental principles of complex numbers, the above expression is equivalent to

$$
2 c_{2}=c_{1}^{2}+y\left(4-c_{1}^{2}\right)
$$

In the same way,

$$
D_{3}=\left|\begin{array}{cccc}
2 & c_{1} & c_{2} & c_{3} \\
\bar{c}_{1} & 2 & c_{1} & c_{2} \\
\bar{c}_{2} & \bar{c}_{1} & 2 & c_{1} \\
\bar{c}_{3} & \bar{c}_{2} & \bar{c}_{1} & 2
\end{array}\right| .
$$

Then $D_{3} \geq 0$ is equivalent to

$$
\left|\left(4 c_{3}-4 c_{1} c_{2}+c_{1}^{3}\right)\left(4-c_{1}^{2}\right)+c_{1}\left(2 c_{2}-c_{1}^{2}\right)^{2}\right| \leq 2\left(4-c_{1}^{2}\right)^{2}-2\left|\left(2 c_{2}-c_{1}^{2}\right)\right|^{2} .
$$

Simplifying relations (7) and (8), we obtain

$$
4 c_{3}=\left\{c_{1}^{3}+2 c_{1}\left(4-c_{1}^{2}\right) y-c_{1}\left(4-c_{1}^{2}\right) y^{2}+2\left(4-c_{1}^{2}\right)\left(1-|y|^{2}\right) \zeta\right\}
$$

for some complex valued $y$ with $|y| \leq 1$ and for some complex valued $\zeta$ with $|\zeta| \leq 1$. To obtain our result, we refer to the classical method devised by Libera and Zlotkiewicz [13], used by several authors in literature.

\section{Main Result}

Theorem 1. If $f(z) \in \mathcal{R}_{p}(\alpha)(0 \leq \alpha<1)$ with $p \in \mathbb{N}$ then

$$
\left|a_{p+1} a_{p+3}-a_{p+2}^{2}\right| \leq\left[\frac{2 p(1-\alpha)}{p+2}\right]^{2}
$$

and the inequality is sharp.

For the function $f(z)=z^{p}+\sum_{n=p+1}^{\infty} a_{n} z^{n} \in \mathcal{R}_{p}(\alpha)$, by virtue of Definition 1 , there exists an analytic function $g \in \mathcal{P}$ in the open unit disc $E$ with $g(0)=1$ and $\operatorname{Re} g(z)>0$ such that

$$
\frac{f^{\prime}(z)-p \alpha z^{p-1}}{p(1-\alpha) z^{p-1}}=g(z) \Leftrightarrow f^{\prime}(z)-p \alpha z^{p-1}=p(1-\alpha) z^{p-1} g(z) .
$$


Replacing $f^{\prime}(z)$ and $g(z)$ with their equivalent series expressions in (10), we have

$$
p z^{p-1}+\sum_{n=p+1}^{\infty} n a_{n} z^{n-1}-p \alpha z^{p-1}=p(1-\alpha) z^{p-1}\left\{1+\sum_{n=1}^{\infty} c_{n} z^{n}\right\} .
$$

Upon simplification, we obtain

$$
\begin{array}{r}
p(1-\alpha) z^{p-1}+(p+1) a_{p+1} z^{p}+(p+2) a_{p+2} z^{p+1}+(p+3) a_{p+3} z^{p+2}+\ldots= \\
=p(1-\alpha) z^{p-1}\left[1+c_{1} z+c_{2} z^{2}+c_{3} z^{3}+\ldots\right] . \quad \text { (11) }
\end{array}
$$

Equating the coefficients of same powers of $z^{p}, z^{p+1}$ and $z^{p+2}$ in (11), we have

$$
a_{p+1}=\frac{p(1-\alpha) c_{1}}{p+1}, \quad a_{p+2}=\frac{p(1-\alpha) c_{2}}{p+2} \quad \text { and } \quad a_{p+3}=\frac{p(1-\alpha) c_{3}}{p+3} .
$$

Substituting the values of $a_{p+1}, a_{p+2}$, and $a_{p+3}$ from (12) in the functional $\left|a_{p+1} a_{p+3}-a_{p+2}^{2}\right|$, after simplifying we get

$$
\begin{gathered}
\left|a_{p+1} a_{p+3}-a_{p+2}^{2}\right|= \\
=\frac{p^{2}(1-\alpha)^{2}}{(p+1)(p+2)^{2}(p+3)}\left|(p+2)^{2} c_{1} c_{3}-(p+1)(p+3) c_{2}^{2}\right| .
\end{gathered}
$$

The above expression is equivalent to

$$
\left|a_{p+1} a_{p+4}-a_{p+2}^{2}\right|=t\left|d_{1} c_{1} c_{3}+d_{2} c_{2}^{2}\right|
$$

where

$$
t=\frac{p^{2}(1-\alpha)^{2}}{(p+1)(p+2)^{2}(p+3)}, d_{1}=(p+2)^{2} \text { and } d_{2}=-(p+1)(p+3) .
$$

Substituting the values of $c_{2}$ and $c_{3}$ from (7) and (9) respectively from Lemma 2 in the expression $\left|d_{1} c_{1} c_{3}+d_{2} c_{2}^{2}\right|$, which is on the right-hand side of (13), we have

$$
\begin{aligned}
\left|d_{1} c_{1} c_{3}+d_{2} c_{2}^{2}\right|= & \mid d_{1} c_{1} \times \frac{1}{4}\left\{c_{1}^{3}+2 c_{1}\left(4-c_{1}^{2}\right) y-c_{1}\left(4-c_{1}^{2}\right) y^{2}+\right. \\
& \left.+2\left(4-c_{1}^{2}\right)\left(1-|y|^{2}\right) \zeta\right\}+d_{2} \times \frac{1}{4}\left\{c_{1}^{2}+y\left(4-c_{1}^{2}\right)\right\}^{2} \mid
\end{aligned}
$$




$$
\begin{aligned}
4\left|d_{1} c_{1} c_{3}+d_{2} c_{2}^{2}\right|=\mid & \left(d_{1}+d_{2}\right) c_{1}^{4}+2 d_{1} c_{1}\left(4-c_{1}^{2}\right) \zeta+2\left(d_{1}+d_{2}\right) c_{1}^{2}\left(4-c_{1}^{2}\right) y- \\
& -\left\{d_{1} c_{1}^{2} y^{2}+2 d_{1} c_{1}|y|^{2} \zeta-d_{2}\left(4-c_{1}^{2}\right) y^{2}\right\}\left(4-c_{1}^{2}\right) \mid ; \\
4\left|d_{1} c_{1} c_{3}+d_{2} c_{2}^{2}\right|=\mid & \left(d_{1}+d_{2}\right) c_{1}^{4}+2 d_{1} c_{1}\left(4-c_{1}^{2}\right) \zeta+2\left(d_{1}+d_{2}\right) c_{1}^{2}\left(4-c_{1}^{2}\right) y- \\
& -\left\{\left(d_{1}+d_{2}\right) c_{1}^{2} y^{2}+2 d_{1} c_{1}|y|^{2} \zeta-4 d_{2} y^{2}\right\}\left(4-c_{1}^{2}\right) \mid .
\end{aligned}
$$

Applying the triangle inequality, we get

$$
\begin{aligned}
4\left|d_{1} c_{1} c_{3}+d_{2} c_{2}^{2}\right| & \leq\left|\left(d_{1}+d_{2}\right) c_{1}^{4}+2 d_{1} c_{1}\left(4-c_{1}^{2}\right)\right| \zeta\left|+2\left(d_{1}+d_{2}\right) c_{1}^{2}\left(4-c_{1}^{2}\right)\right| y \mid+ \\
& +\left\{\left(d_{1}+d_{2}\right) c_{1}^{2}|y|^{2}+2 d_{1} c_{1}|y|^{2}|\zeta|-4 d_{2}|y|^{2}\right\}\left(4-c_{1}^{2}\right) \mid .
\end{aligned}
$$

Using the fact that $|\zeta|<1$ in the above iequality, we obtain

$$
\begin{aligned}
4\left|d_{1} c_{1} c_{3}+d_{2} c_{2}^{2}\right| \leq \mid & \left(d_{1}+d_{2}\right) c_{1}^{4}+2 d_{1} c_{1}\left(4-c_{1}^{2}\right)+2\left(d_{1}+d_{2}\right) c_{1}^{2}\left(4-c_{1}^{2}\right)|y|+ \\
+ & \left\{\left(d_{1}+d_{2}\right) c_{1}^{2}+2 d_{1} c_{1}-4 d_{2}\right\}\left(4-c_{1}^{2}\right)|y|^{2} \mid .
\end{aligned}
$$

Using the values of $d_{1}, d_{2}$ given in (14), we can write

$$
\begin{gathered}
d_{1}+d_{2}=1 \text { and }\left\{\left(d_{1}+d_{2}\right) c_{1}^{2}+2 d_{1} c_{1}-4 d_{2}\right\}= \\
=c_{1}^{2}+2(p+2)^{2} c_{1}+4(p+1)(p+3) .
\end{gathered}
$$

Substituting the values from (16) and value of $d_{1}$ from (14) to the righthand side of (15), we have

$$
\begin{aligned}
4\left|d_{1} c_{1} c_{3}+d_{2} c_{2}^{2}\right| & \leq\left|c_{1}^{4}+2(p+2)^{2} c_{1}\left(4-c_{1}^{2}\right)+2 c_{1}^{2}\left(4-c_{1}^{2}\right)\right| y \mid+ \\
+ & \left\{c_{1}^{2}+2(p+2)^{2} c_{1}+4(p+1)(p+3)\right\}\left(4-c_{1}^{2}\right)|y|^{2} \mid
\end{aligned}
$$

Consider $\quad\left\{c_{1}^{2}+2(p+2)^{2} c_{1}+4(p+1)(p+3)\right\}=$

$$
\begin{aligned}
&=\left[\left\{c_{1}+(p+2)^{2}\right\}^{2}-\left(\sqrt{p^{4}+8 p^{3}+20 p^{2}+16 p+4}\right)^{2}\right]= \\
&=\left[c_{1}+\right.\left.\left\{(p+2)^{2}+\left(\sqrt{p^{4}+8 p^{3}+20 p^{2}+16 p+4}\right)\right\}\right] \times \\
& \times\left[c_{1}+\left\{(p+2)^{2}-\left(\sqrt{p^{4}+8 p^{3}+20 p^{2}+16 p+4}\right)\right\}\right] .
\end{aligned}
$$


Noting that $\left(c_{1}+a\right)\left(c_{1}+b\right) \geq\left(c_{1}-a\right)\left(c_{1}-b\right)$, where $a, b \geq 0$, and $c_{1} \in[0,2]$ in the above expression, we obtain

$$
\begin{aligned}
\left\{c_{1}^{2}+2(p+2)^{2} c_{1}+4(p+1)\right. & (p+3)\} \\
& \geq\left\{c_{1}^{2}-2(p+2)^{2} c_{1}+4(p+1)(p+3)\right\} .
\end{aligned}
$$

From expressions (17) and (18), we get

$$
\begin{aligned}
4\left|d_{1} c_{1} c_{3}+d_{2} c_{2}^{2}\right| \leq\left|c_{1}^{4}+2(p+2)^{2} c_{1}\left(4-c_{1}^{2}\right)+2 c_{1}^{2}\left(4-c_{1}^{2}\right)\right| y \mid+ \\
+\left\{c_{1}^{2}-2(p+2)^{2} c_{1}+4(p+1)(p+3)\right\}\left(4-c_{1}^{2}\right)|y|^{2} \mid
\end{aligned}
$$

Choosing $c_{1}=c \in[0,2]$, replacing $|y|$ by $\mu$ on the right-hand side of (19), we obtain

$$
\begin{gathered}
4\left|d_{1} c_{1} c_{3}+d_{2} c_{2}^{2}\right| \leq\left[c^{4}+2(p+2)^{2} c\left(4-c^{2}\right)+2 c^{2}\left(4-c^{2}\right) \mu+\right. \\
\left.+\left\{c^{2}-2(p+2)^{2} c+4(p+1)(p+3)\right\}\left(4-c^{2}\right) \mu^{2}\right]= \\
=F(c, \mu), 0 \leq \mu=|y| \leq 1 \text { and } 0 \leq c \leq 2 .
\end{gathered}
$$

Next, we maximize function $F(c, \mu)$ on the closed region $[0,2] \times[0,1]$. Differentiating $F(c, \mu)$ given in the right-hand side of (20) partially with respect to $\mu$, we get

$$
\frac{\partial F}{\partial \mu}=2\left[c^{2}+\left\{c^{2}-2(p+2)^{2} c+4(p+1)(p+3)\right\} \mu\right]\left(4-c^{2}\right) .
$$

For $0<\mu<1$, for fixed $c$ with $0<c<2$ and $p \in \mathbb{N}$, from (21), we observe that $\frac{\partial F}{\partial \mu}>0$. Therefore, $F(c, \mu)$ becomes an increasing function of $\mu$ and hence it cannot have a maximum value at any point in the interior of the closed region $[0,2] \times[0,1]$. The maximum value of $F(c, \mu)$ occurs on the boundary i.e., when $\mu=1$. Therefore, for fixed $c \in[0,2]$, we have

$$
\max _{0 \leq \mu \leq 1} F(c, \mu)=F(c, 1)=G(c) .
$$

Replacing $\mu$ by 1 in $F(c, \mu)$, it simplifies to

$$
\begin{gathered}
G(c)=-2 c^{4}-4 p(p+4) c^{2}+16(p+1)(p+3), \\
G^{\prime}(c)=-8 c^{3}-8 p(p+4) c .
\end{gathered}
$$


From (24), we observe that $G^{\prime}(c) \leq 0$ for every $c \in[0,2]$ with $p \in \mathbb{N}$. Consequently, $G(c)$ becomes a decreasing function of $c$, whose maximum value occurs at $c=0$ only. From (23), the maximum value of $G(c)$ at $c=0$ is obtained to be

$$
G_{\max }=G(0)=16(p+1)(p+3) .
$$

Simplifying expressions (20) and (25), we get

$$
\left|d_{1} c_{1} c_{3}+d_{2} c_{2}^{2}\right| \leq 4(p+1)(p+3) .
$$

From relations (13) and (26), along with the value of $t$ in (14), upon simplification, we obtain

$$
\left|a_{p+1} a_{p+3}-a_{p+2}^{2}\right| \leq\left[\frac{2 p(1-\alpha)}{p+2}\right]^{2} .
$$

By setting $c_{1}=c=0$ and selecting $y=1$ in the expressions (7) and (9), we find that $c_{2}=2$ and $c_{3}=0$, respectively. Substituting the values $c_{2}=2, c_{3}=0$, and $d_{2}=-(p+1)(p+3)$ in $(26)$, we observe that equality is attained, which shows that our result is sharp. For the values $c_{2}=2$ and $c_{3}=0$, from (6) we derive the extremal function given by

$$
g(z)=1+2 z^{2}+2 z^{4}+\ldots=\frac{1+z^{2}}{1-z^{2}} .
$$

So that from (10), we have

$$
\frac{f^{\prime}(z)-p \alpha z^{p-1}}{p(1-\alpha) z^{p-1}}=1+2 z^{2}+2 z^{4}+\ldots=\frac{1+z^{2}}{1-z^{2}} .
$$

This completes the proof of our Theorem.

Remark 1. If $p=1$ and $\alpha=0$ in (27) then $\left|a_{2} a_{4}-a_{3}^{2}\right| \leq \frac{4}{9}$; this coincides with the result of Janteng et al. [7].

Remark 2. If $p=1$ in (27) then $\left|a_{2} a_{4}-a_{3}^{2}\right| \leq \frac{4(1-\alpha)^{2}}{9}$, this result is same as that of Vamshee Krishna and RamReddy [14].

Remark 3. If $\alpha=0$ in (27) then $\left|a_{p+1} a_{p+3}-a_{p+2}^{2}\right| \leq\left[\frac{2 p}{p+2}\right]^{2}$, this result coincides with the result obtained by Vamshee Krishna and RamReddy [15]. 
Acknowledgment. The authors express sincere thanks to the esteemed Referee(s) for their careful readings, valuable suggestions and comments, which helped to improve the paper.

\section{References}

[1] Louis de Branges de Bourcia A proof of Bieberbach conjecture. Acta Mathematica, 1985, vol. 154, no. 1-2, pp. 137-152.

[2] Pommerenke Ch. On the coefficients and Hankel determinants of univalent functions. J. Lond. Math. Soc., 1966, vol. 41, pp. 111-122.

[3] Noonan J. W., Thomas D. K. On the second Hankel determinant of areally mean p-valent functions. Trans. Amer. Math. Soc., 1976, vol. 223, no. 2, pp. 337-346.

[4] Noor K. I. Hankel determinant problem for the class of functions with bounded boundary rotation. Rev. Roumaine Math. Pures Appl., 1983, vol. 28 , no. 8 , pp. $731-739$.

[5] Layman J. W. The Hankel transform and some of its properties J. Integer Seq., 2001, vol. 4, no. 1, pp. 1-11.

[6] Ali R. M. Coefficients of the inverse of strongly starlike functions. Bull. Malays. Math. Sci. Soc., (second series), 2003, vol. 26, no. 1, pp. 63-71.

[7] Janteng A., Halim S. A., Darus M. Hankel Determinant for starlike and convex functions. Int. J. Math. Anal. (Ruse), 2007, vol. 1, no. 13, pp. 619625 .

[8] MacGregor T. H. Functions whose derivative have a positive real part. Trans. Amer. Math. Soc., 1962, vol. 104, no. 3, pp. 532-537.

[9] Duren P. L. Univalent functions. vol. 259 of Grundlehren der Mathematischen Wissenschaften, Springer, New York, USA, 1983.

[10] Pommerenke Ch. Univalent functions. Gottingen: Vandenhoeck and Ruprecht, 1975.

[11] Simon B. Orthogonal polynomials on the unit circle, part 1. Classical theo$r y$. vol. 54, American mathematical society colloquium publications. Providence (RI): American Mathematical Society; 2005.

[12] Grenander U., Szegö G. Toeplitz forms and their applications. 2nd ed. New York (NY): Chelsea Publishing Co.; 1984.

[13] Libera R. J., Zlotkiewicz E. J. Coefficient bounds for the inverse of a function with derivative in $\mathcal{P}$, Proc. Amer. Math. Soc., 1983, vol. 87, pp. 251257. 
[14] Vamshee Krishna D., RamReddy T. Coefficient inequality for a function whose derivative has a positive real part of order alpha. Mathematica Bohemica, 2015, vol. 140, no. 1, pp. 43-52.

[15] Vamshee Krishna D., RamReddy T. Coefficient inequality for certain pvalent analytic functions. Rocky Mountain J. Math., 2014, vol. 44, no. 6, pp. 1941-1959.

Received January 10, 2016.

In revised form, July 03, 2016.

Accepted July 03, 2016.

GIT, GITAM University

Visakhapatnam 530 045, A. P., India

E-mail: vamsheekrishna1972@gmail.com

Kakatiya University

Warangal 506 009, T. S., India

E-mail: reddytr2@gmail.com 